\title{
Asimetrías faciales y maloclusiones en pacientes con tortícolis muscular congénita. Una revisión sistemática
}

\author{
Facial asymmetries and dental malocclusions in patients with congenital \\ muscular torticollis. One review systematic
}

\author{
CUETO BLANCO S* \\ PIPA VALLEJO A** \\ GONZÁLEZ GARCÍA M*** \\ PIPA MUÑIZ M $\mathbf{1} * * * *$ \\ PIPA MUÑIZ C*****
}

\begin{abstract}
Cueto Blanco S, Pipa Vallejo A, González García M, Pipa Muñiz M, Pipa Muñiz C. Asimetrías faciales y maloclusiones en pacientes con tortícolis muscular congénita. Una revisión sistemática. Av Periodon Implantol. 2015; 27, 1: 11-18.
\end{abstract}

\section{RESUMEN}

Las deformidades dentofaciales generan déficits funcionales y estéticos. Cuando la tortícolis muscular congénita (TMC) no se detecta precozmente y, por tanto, no se trata, pueden establecerse alteraciones craneofaciales (asimetrías, desviación lateral mandibular, deformación plagiocefálica del frontal, retracción del cigoma, distopia orbitaria, disminución de la dimensión vertical del lado afectado, desplazamiento posterior del oído del lado afectado, inclinación del plano comisural) y oclusodentales (inclinación del plano oclusal, mordida cruzada unilateral en el lado afecto, desviación de la línea media dentaria hacia el lado afectado).

Es preciso, por tanto, un diagnóstico precoz, durante las primeras semanas de vida, para que esta anomalía pueda corregirse instaurando un tratamiento conservador mediante fisioterapia. Los casos que no responden a este tipo de tratamiento o que han tardado en diagnosticarse muy probablemente precisaran tratamiento quirúrgico.

El propósito de este artículo es hacer una revisión sistemática de la tortícolis muscular congénita, centrándose fundamentalmente en la importancia del diagnóstico temprano y en las anomalías craneofaciales fundamentalmente, y oclusales que se producen si no se corrige.

PALABRAS CLAVE: Terapia anticoagulante, hemostasia, protocolo, extracción dental.

\section{SUMIMARY}

Dentofacial deformities generate functional and aesthetic deficits. When congenital muscular torticollisis not detected, craniofacial alterations (asymmetries, mandible lateral deviation, frontal deformational plagiocephaly, recession of the ipsilateral zygoma, orbital dystopia, reduction of vertical facial height on the affected side, posterior displacement of the ipsilateral ear, commisural canting) and dental malocclusions (occlusal plane canting, unilateral crossbite in the affected side, deviation of the lower center line to the affected side) can be established.

Early diagnosis is necessary during the first weeks of life, to correct this anomaly by a conservative treatment with physical therapy. Cases that do not respond to this treatment or who have been diagnosed later will probably require surgical treatment.

\footnotetext{
* Odontóloga. Máster en Ortodoncia. Escuela de Odontología. Facultad de Medicina. Universidad de Oviedo.

** Profesor Asociado. Escuela de Odontología. Facultad de Medicina. Universidad de Oviedo.

*** Profesor Asociado. Servicio de Cirugía Oral y Maxilofacial del HUCA.

**** Médico. Especialista en Aparato Digestivo. Hospital de Cabueñes.

***** Profesora Asociada. Escuela de Odontología. Facultad de Medicina. Universidad de Oviedo.
} 
The aim of this article is to make asystematic review of the congenital muscular torticollis, focusing on the importance of early diagnosis and in the craniofacial and oclusal anomalies that occur if the congenital muscular torticollis is untreated.

KEY WORDS: Torticollis, congenital, botulinum toxin, craniofacial deformation.

Fecha de recepción: 22 de diciembre de 2014.

Fecha de aceptación: 15 de enero de 2015.

\section{INTRODUCCIÓN}

Existen anomalías congénitas musculoesqueléticas que pueden dar lugar a alteraciones en la estética y funcionalidad facial y por ende en la vida de relación y social que condiciona de forma dramática la actividad diaria de estos pacientes afectos de este tipo de patología.

Entre un 5 y un 12\% de la población presentan una deformidad dentofacial que va a determinar una deficiencia funcional y estética.

Esta deformidad dentofacial puede limitar, y en no pocos casos condicionar, de manera determinante, actividades habituales y fundamentales de la vida diaria como la fonación y la masticación y generar dolor e incapacidad funcional, fundamentalmente en la articulación temporomandibular, problemas que se pueden considerar incapacitantes, desde el punto de vista físico, así como condicionantes tanto emocional como socialmente.

Asimetrías craneales y faciales a menudo acompañan a la tortícolis muscular congénita, pero rara vez se ven cuando la tortícolis es adquirida después del nacimiento.

La palabra tortícolis fue acuñada por François Rebelais en 1532, que la formó del latín tortum (torcido) y collum (cuello): cuello torcido.

Es la tercera patología congénita más frecuente después de la luxación de cadera y el pie zambo.

La base etiológica de la tortícolis muscular congénita parece residir en un desorden del desarrollo del músculo esternocleidomastoideo (MECM) queda como resultado el acortamiento y fibrosis del mismo (1) y, por lo tanto, se va a caracterizar, fundamentalmente, por inclinación de la cabeza hacia el lado afecto y rotación de la cara hacia el lado contrario (2) (Figs. 1 y 2).

La incidencia de TMC es entre un 0,4 y un $1,9 \%$ de los recién nacidos y la etiopatogenia es muy contro- vertida, se cree que podría estar causada por traumas durante el nacimiento, partos difíciles, presentación de nalgas, cesáreas o usos de fórceps, aunque otros autores apuntan únicamente a factores prenatales (3-6).

\section{DIAGNÓSTICO DE LA TORTÍCOLIS MUSCULAR CONGENITA}

Para obtener buenos resultados y evitar complicaciones y complejas correcciones quirúrgicas es muy importante un diagnóstico precoz de la TMC, por lo tanto, debería establecerse en los primeros meses de vida (7).

Para ello es preciso realizar una historia clínica completa en la que se recojan las dificultades durante el parto o la presentación de nalgas y una exploración física para valorar el rango de movilidad de la cabeza y el cuello, dado que durante las primeras semanas de vida se evidencia una retracción del músculo esternocleidomastoideo, asociado o no a un tumor palpable, responsable de la asimetría y del déficit de movilidad de la cabeza y el cuello $(2,8)$.

La ultrasonografía es la prueba diagnóstica que se usa habitualmente para confirmar la existencia de la masa en el cuello o la hipertrofia del MECM (9).

Hay que tener en cuenta, así mismo, que el $12 \%$ de los pacientes diagnosticados de TMC pueden presentar displasia de cadera, por lo que deberían valorarse pruebas como la radiografía anteroposterior de pelvis en niños con TMC mayores de 6 meses y un estudio con ultrasonidos en los menores de 6 meses (10).

Es imprescindible, por otro lado, para evitar posibles errores en el diagnóstico, realizar un diagnóstico diferencial entre la tortícolis muscular congénita y otras formas de tortícolis (Tabla 1).

Los pacientes que no han sido diagnosticados a una edad temprana, pueden presentar en mayor o menor 


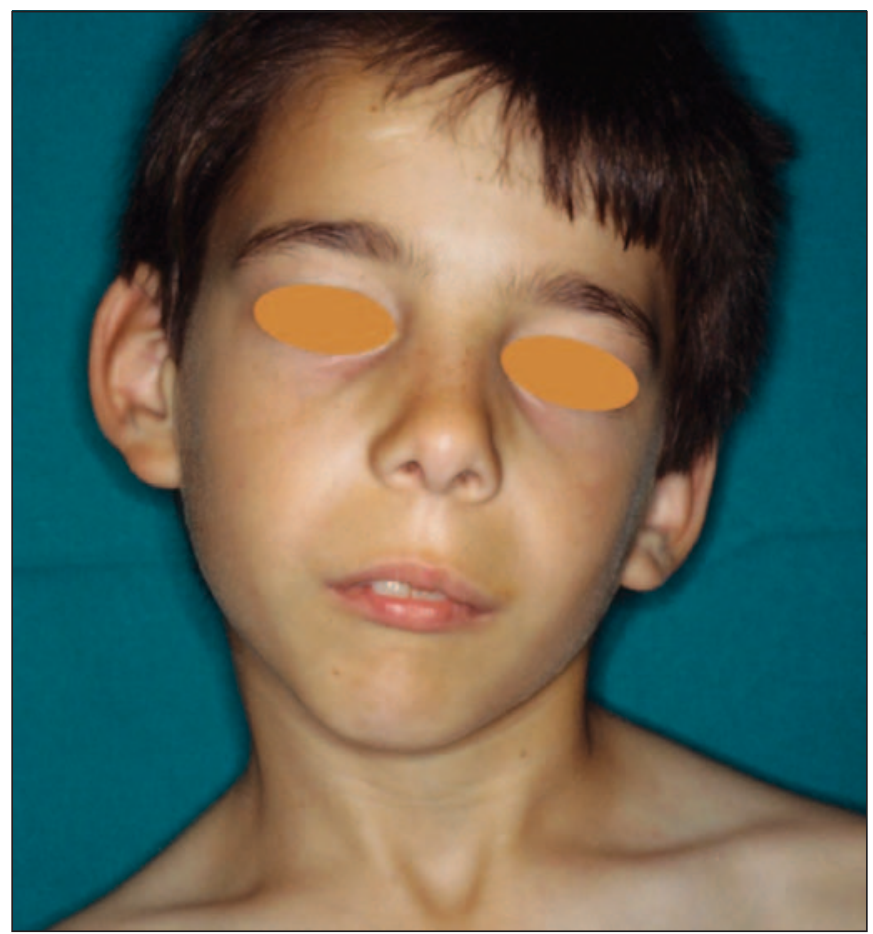

Fig. 1.

\section{TABLA 1.- OTRAS FORIMASS DE TORTÍCOLIS (ADAPTADA DE MOHAN)}

\section{- Condiciones oculares:}

- Parálisis del músculo oblicuo superior.

Parálisis del músculo recto lateral.

- Nistagmus.

\section{- Anomalías de la columna cervical:}

- S. Klippel-Feil.

- Subluxación de Cl-C2.

- Hemiatlas.

- Fusión ósea cervical.

\section{- Infecciones:}

- Absceso parafaríngeo.

- Sinusitis.

- Infecciones de vía respiratoria alta.

- Trauma.

- Terapia de radiación.

- Tumores a nivel del sistema nervioso central (especialmente fosa posterior).

- Parálisis del plexo braquial.

- Tortícolis paroxística benigna.

- Síndrome de Sandifer (tortícolis y reflujo gastroesofágico).

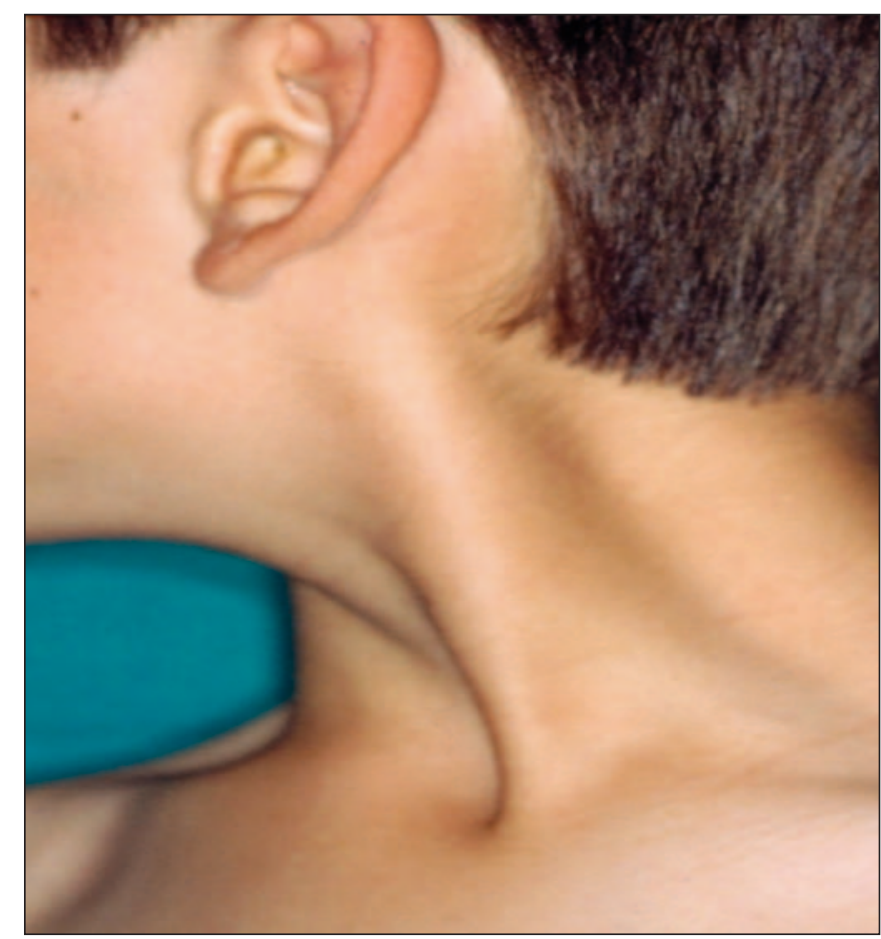

Fig. 2.

medida las alteraciones y asimetrías craneofaciales que se describen en el siguiente apartado y precisarán de un estudio radiológico que incluya telerradiografía lateral y anteroposterior de cráneo, radiografías cervicales e incluso TAC (11). También es necesario un estudio cefalométrico para la determinación y el seguimiento de la asimetría (12). Con los avances en las técnicas de imagen, hoy en día es común el uso de la tomografía computarizada 3D $(13,14)$.

\section{ALTERACIONES CRANEOFACIALESY OCLUSALES EN PACIENTES NO DIAGNOSTICADOS}

Si la TMC no se detecta pronto y, por lo tanto, no se trata, es frecuente que durante el crecimiento se haga evidente una asimetría craneofacial, deformación frontal plagiocefálica, con retrusión del cigoma y del frente ipsilateral, reducción de la altura facial vertical del lado afecto, distopia orbitaria (el ojo del lado no afectado está más alto) y desplazamiento posterior del oído del lado afectado. También se ha descrito inclinación de la comisura hacia el lado afecto e inclinación del plano oclusal. En estos casos, el plano oclusal y el plano orbital no son paralelos, sino que están más próxi- 
mos en el lado afectado de la cara). Puede aparecer también reducción de la apertura palpebral en el lado afectado y desviación de la punta de la nariz hacia el lado afectado (13-16).

La figura 3 sintetiza de manera evidente estos cambios óseos y faciales.

La TMC también puede ser con relativa frecuencia causa de desviación lateral de la mandíbula. En tortícolis leves la asimetría mandibular puede pasar inadvertida hasta la maduración esquelética cuando la mandíbula finaliza su crecimiento. Ante la asimetría mandibular es preciso realizar un diagnóstico diferencial con otras causas de desviación mandibular lateral como la fractura condilar, la hiperplasia condilar, la artritis juvenil condilar, la microsomía hemifacial, la craneosinostosis coronal unilateral y la deformación plagiocefálica (17) (Tabla 2).

La asimetría craneal ya ha sido determinada en pacientes menores de 6 meses (13). Para otros autores

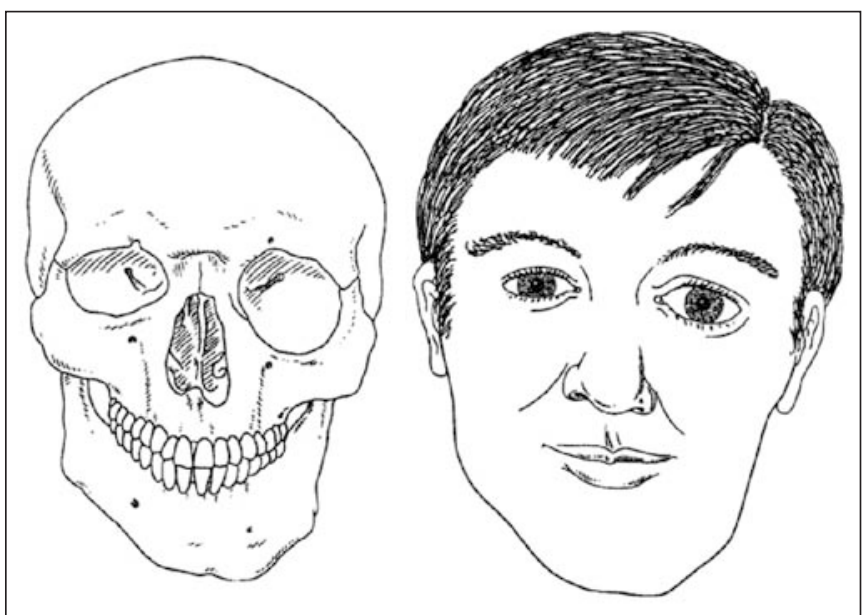

Fig. 3: Esquema de los cambios observados en casos avanzados de tortícolis (tomada de Hollier).

en pacientes con TMC sin tratar, la deformidad y la asimetría ósea comienza a hacerse evidente a los 5 años, edad a la que empieza a observarse la anorma-

\section{TABLA 2.- DIAGNÓSTICO DIFERENCIAL DE LA DESVIACIÓN MANDIBULAR IDIOPÁTICA (KAWAMOTO)}

\begin{tabular}{|l|l|l|}
\hline Causa & Anormalidad & Hallazgos diagnósticos \\
\hline Tortícolis muscular & Deformación base craneal & $\begin{array}{l}\text { Inclinación de la cabeza y rotación restringida del } \\
\text { cuello. }\end{array}$ \\
\hline Fractura condilar & Anormalidad condilar & $\begin{array}{l}\text { CT scan: cuello condilar corto, fosa glenoidea flat, } \\
\text { ángulo condilar irregular. }\end{array}$ \\
\hline Hiperplasia condilar & $\begin{array}{l}\text { Anormalidad condilar } \\
\text { condilar. }\end{array}$ & Panorámica: altura vertical incrementada del cuello \\
\hline Artritis condilar juvenil & Anormalidad condilar & $\begin{array}{l}\text { Radiografía: Espacio ATM estrechado, osteofitos. } \\
\text { Resonancia magnética: erosión cartílago condilar. }\end{array}$ \\
\hline Microsomía hemifacial & Anormalidad condilar & $\begin{array}{l}\text { Hallazgos variables, canteo oclusal. } \\
\text { Panorámica: cóndilo/rama ausente o hipoplásico. } \\
\text { Tejidos blandos: microtia, macrostomía. }\end{array}$ \\
\hline $\begin{array}{l}\text { Craneosinostosis } \\
\text { coronal unilateral }\end{array}$ & Deformación base craneal & $\begin{array}{l}\text { Punto de barbilla contralateral y raíz nasal ipsilateral } \\
\text { desde sinostosis. }\end{array}$ \\
\hline $\begin{array}{l}\text { Deformación } \\
\text { plagiocefálica }\end{array}$ & Deformación base craneal & $\begin{array}{l}\text { Forma de paralelogramo de la cabeza con oídos } \\
\text { (base craneal y fosa glenoidea) anteriormente } \\
\text { posicionado en lado afectado. }\end{array}$ \\
\hline
\end{tabular}


lidad mandibular y la inclinación del plano oclusal. Más adelante aparecen las anormalidades del maxilar y finalmente las de la órbita. En pacientes con más edad es obvia la restricción del desarrollo óseo facial del lado ipsilateral con disminución de las alturas maxilares y orbitarias, así como el estrechamiento de la parte inferior de la hemifacies del lado de la lesión.

La restricción del desarrollo óseo facial ipsilateral está causada por la limitación de la movilidad del MECM (acortado y fibrótico). La deformación contralateral se ve influenciada por un crecimiento compensatorio, creando una apariencia facial curvilínea desde una vista frontal. El plano oclusal está inclinado debido al desarrollo asimétrico de los huesos faciales (14).

A nivel intraoral estos pacientes con tortícolis muscular congénita, pueden presentar desviación de la línea media dentaria inferior hacia el lado afectado, mordida cruzada unilateral en ese mismo lado, así como una tendencia a la relación molar de clase II en ese lado afecto y relación de clase III en el lado contrario $(3,16,18,19)$.

La figura 4, tomada de Chate, muestra las alteraciones oclusales que se pueden observar en estos casos.
También se puede observar en casos de tortícolis muscular congénita la presencia de un acusado resalte que es frecuente en este tipo de patologías (Figura 5).

\section{TRATAMIENTO}

En muchos casos, los tumores del MECM se resuelven espontáneamente sin dejar ningún tipo de déficit resi-

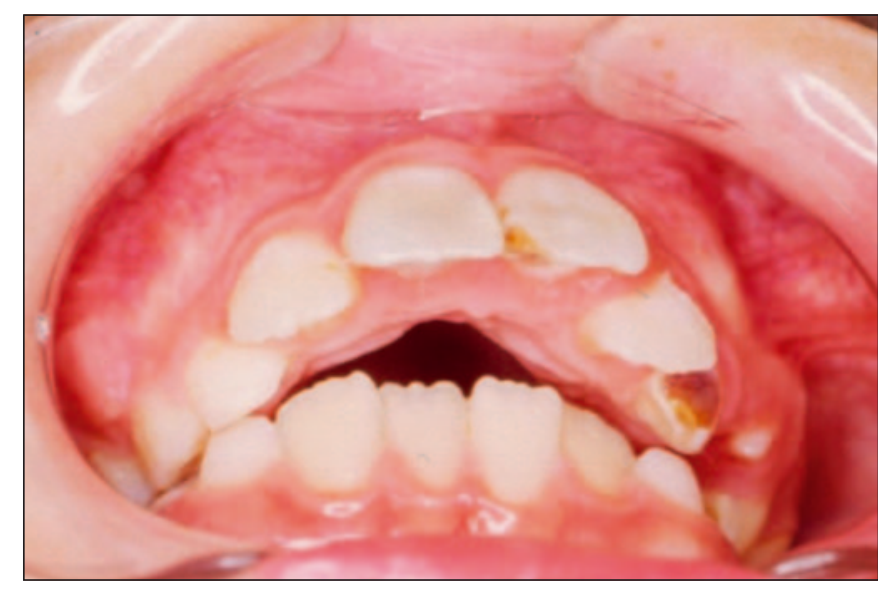

Fig. 5.

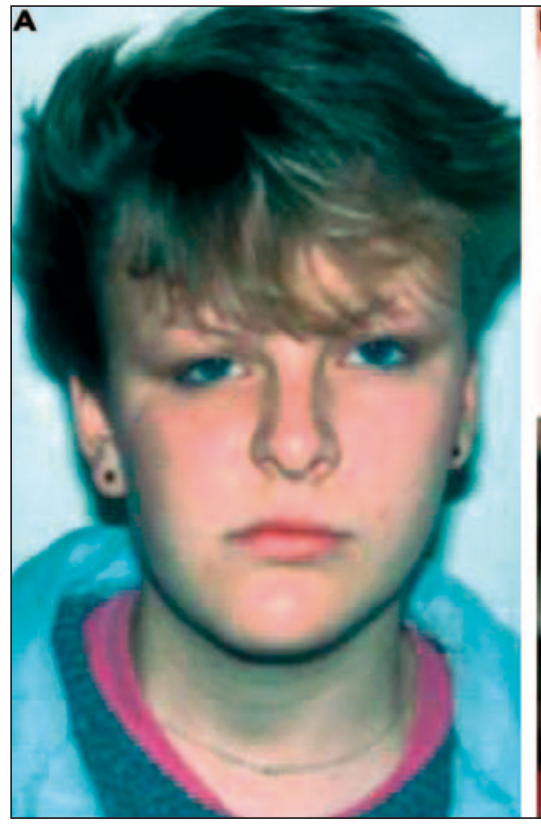

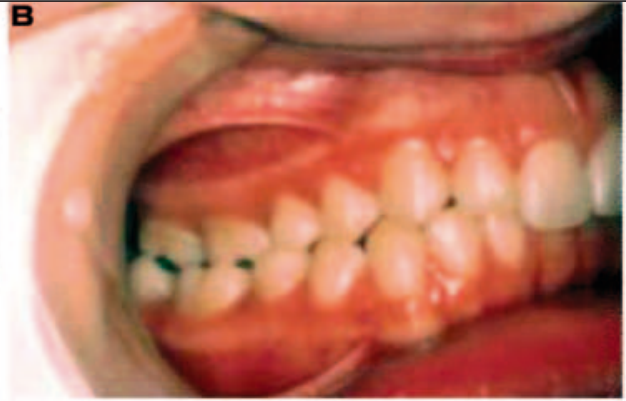

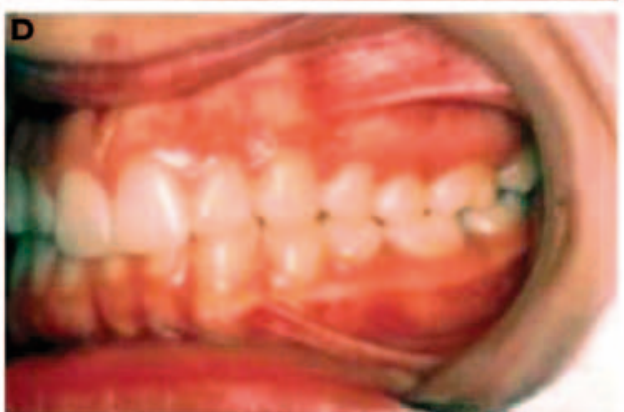

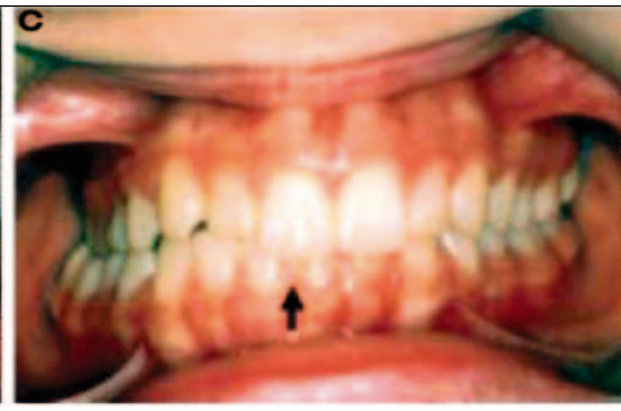

Fig. 4. 
dual (2). Si no es así, se establece un tratamiento conservador mediante fisioterapia que incluye ejercicios manuales de estiramientos, masajes, ejercicios de fortalecimiento, ultrasonografía terapéutica (9) e incluso el uso de cascos adaptados (20).

Los niños pequeños, especialmente aquellos menores de 1 mes de vida, necesitan menor tiempo de tratamiento con mejores resultados (7).

De hecho, cuanto antes sean diagnosticados estos niños con tortícolis, antes se comienza con el tratamiento conservador, la duración del tratamiento rehabilitador es menor.

La mayor o menor limitación del movimiento cervical es un factor de pronóstico de los resultados de la rehabilitación en bebes con TMC, ya que una mayor limitación de la movilidad cervical antes de comenzar el tratamiento conservador está correlacionado con la mayor duración del tratamiento (9).

Aunque para casos resistentes, o en aquellos tratados después del año de edad, es necesario el alargamiento quirúrgico del músculo o la liberación del músculo esternocleidomastoideo (21), se han descrito casos de niños en los que tras no responder al tratamiento conservador se ha optado por un tratamiento mediante inyección de toxina botulínica en el músculo ECM afectado junto con fisioterapia posterior, consiguiendo resultados tan satisfactorios como para poder descartar la opción quirúrgica (20). De hecho, en pacientes adultos hay autores que recomiendan intentar tratarlos con inyecciones de toxina botulínica justo por detrás del MECM antes de considerar la opción quirúrgica $(22,23)$.

El mayor beneficio con el tratamiento quirúrgico se logra en edades comprendidas entre 1 y 4 años, pero se pueden favorecer del tratamiento pacientes de 6 años (12).

Se han obtenido resultados relativamente satisfactorios en pacientes mayores de 10 años (entre 10 y 19 años) tras ser intervenidos quirúrgicamente mediante liberación bipolar del MECM junto con un postoperatorio que incluía tracción de la cabeza durante 3 semanas seguidos de fisioterapia (24), así como de una paciente de 18 años tras ser sometida a liberación unipolar del MECM, corsé cervical al $2^{\circ}$ día de la operación para mantener el cuello en una posición anatómica normal y fisioterapia a partir del $5^{\circ}$ día y durante 2 meses. Los resultados funcionales fueron mejores que los estéticos con una mejora de la inclinación de la cabeza y de la movilidad cervical , mientras que la asimetría facial fue más resistente al cambio (3). Incluso se ha descrito un caso de paciente adulto de 28 años de edad, sometido a resección unipolar del MECM en el extremo distal con resultados satisfactorios, restaurando el rango de movimiento del cuello, resolviendo la inclinación de la cabeza y mejorando la calidad de vida del paciente que es muy importante (25).

Las mejoras en la deformidad y la asimetría craneofacial tras la liberación quirúrgica del MECM, son mejores en pacientes sometidos a cirugía antes de los 5 años de edad que en pacientes tratados en edades posteriores. Los cambios posquirúrgicos en cuanto a la deformidad craneofacial ocurren con más frecuencia durante el primer año del postoperatorio (26).

La deformación plagiocefálica es más difícil de corregir tras la pubertad por la falta de potencial para el crecimiento y posibilidad del remodelado (3).

Sin embargo, otros autores son partidarios de que el momento quirúrgico no sea determinado por la edad sino en función del grado de madurez del paciente para que sea capaz de realizar satisfactoriamente el programa de ejercicios y tracciones.

Comparando dos grupos de pacientes, unos operados entre el primer año y los 4 años de edad y otros entre los 5 y los 16 años de edad, no se encontraron diferencias en cuanto a asimetría craneofacial y contractura residual y encontraron mejores resultados en cuanto a movilidad, cicatrización quirúrgica y grado de inclinación de la cabeza (27).

\section{MATERIAL Y MÉTODOS}

Se realiza una búsqueda electrónica exhaustiva de publicaciones o artículos en relación con la tortícolis muscular congénita entre los años 1994 y 2014, fundamentada casi en su totalidad en la base de datos PubMed.

De los 174 artículos encontrados se hace una selección de los relacionados sobretodo con asimetrías y deformidades dentofaciales, los que están en relación con las distintas opciones terapéuticas actuales y los de especial interés respecto a la tortícolis muscular congénita, apareciendo un total de 25 artículos dentro de esa base de datos. 


\section{DISCUSIÓN}

Es necesario hacer hincapié en este tipo de patología para que pueda ser detectada por los pediatras o los padres lo antes posible, de forma precoz y pueda resolverse de una manera sencilla y rápida mediante terapia física y evitar retrasos en el diagnóstico que puedan oscurecer el abordaje terapéutico y comprometer una evolución a priori favorable.

Los casos que no se diagnostican con prontitud desarrollan con el crecimiento deformaciones y asimetrías craneofaciales que incluyen deformación plagiocefálica, retrusión del cigoma, distopia orbitaria (15), desviación lateral de la mandíbula (17), alteración del plano orbitario y oclusal (14) y maloclusiones dentarias $(3,16,18,19)$.

Se ha establecido que la deformidad y la asimetría ósea comienzan a hacerse evidentes a partir de los 5 años de edad (14), sin embargo, otros autores han determinado asimetría craneal en pacientes menores de 6 meses (13).

Un diagnóstico temprano y por tanto un inicio precoz en el tratamiento conservador son factores de buen pronóstico. Se ha observado que los bebés menores de 1 mes de vida necesitan menor tiempo de tratamiento con mejores resultados (7).

De hecho, se ha demostrado que la duración del tratamiento rehabilitador es menor cuando antes se inicie. Otro factor de pronóstico importante es el rango de movilidad cervical, dado que los pacientes con mayor limitación de la movilidad cervical precisan mayor duración del tratamiento (9).

En casos de tortícolis muscular congénita recalcitrante que no responde al tratamiento conservador, el momento del tratamiento quirúrgico es controvertido ya que muchos autores coinciden en que la asimetría es progresiva si no se trata y en que la severidad de las deformaciones por tanto aumenta con la edad $(13,14)$.

Se han conseguido mayores mejoras en cuanto a corrección de la deformidad y la asimetría ósea en los pacientes intervenidos quirúrgicamente antes de los 5 años de edad (26), además la deformación plagiocefálica es más difícil de corregir tras la pubertad por la falta de potencial para el crecimiento y remodelado óseo (3). Sin embargo otros autores son partidarios de que el momento quirúrgico no sea determinado por la edad sino por el grado de madurez del paciente para que sea capaz de realizar satisfactoriamente el programa de ejercicios posquirúrgicos, encontrando mejores resultados en cuanto a movilidad, cicatrización quirúrgica y grado de inclinación de la cabeza (27).

Otra opción terapéutica que se contempla antes del tratamiento quirúrgico es la inyección del MECM con toxina botulínica $(20,22,23)$.

\section{CONCLUSIÓN}

Es, por lo tanto, esencial un diagnóstico precoz de la tortícolis muscular congénita para comenzar con el tratamiento conservador de forma temprana y así evitar que empiecen a establecerse las asimetrías craneofaciales; si no es posible este diagnóstico, o no se consigue corregir esta patología, pueden valorarse otras opciones terapéuticas.

En el caso de requerir cirugía, el momento ideal de tratamiento quirúrgico es más discutible y comprometido y con un pronóstico incierto.

\section{BIBLIOGRAFÍA}

1. Yim SY, Yoon D, Park MC, Lee IJ, Kim JH, Lee MA et al. Integrative analysis of congenital muscular torticollis: from gene expression to clinical significance. BMC Med Genomics 2013,6 (Suppl 2):S10.

2. Do TT. Congenital muscular torticollis: current concepts and review of treatment. Curr Opin Pediatr 2006;18(1): 26-9.

3. Mohan M, Bhat S, Prasad R, Sharma SM, Jain TL. Congenital muscular torticollis-case report and an effective treatment plan.J Maxillofac Oral Surg 2012;11 (3):364-7.

4. Cheng JC, Au AW. Infantile torticollis: a review of 624 cases. J Pediatr Orthop 1994;14(6):802-8.

5. Ho BC, Lee EH, Singh K. Epidemiology, presentation and management of congenital muscular torticollis. Singapore Med J 1999;40(11):675-9.

6. Lee SJ, Han JD, Lee HB, Hwang JH, Kim SY, Park MC et al. Comparison of clinical severity of congenital muscular torticollis based on the method of child birth. Ann Rehabil Med 2011;35(5):641-7. 
7. Petronic I, Brdar R, Cirovic D, Nikolic D, Lukac M, Janic $\mathrm{D}$ et al. Congenital muscular torticollis in children: distribution, treatment duration and out come. Eur J Phys Rehabil Med 2010;46(2):153-8.

8. Peyrou P, Moulies D. Torticollis in children: diagnostic approach. Arch Pediatr 2007;14(10):1264-70.

9. Lee JY, Koh SE, Lee IS, Jung H, Lee J, Kang JI et al. The cervical range of motion as a factor affecting outcome in patients with congenital muscular torticollis. Ann Rehabil Med 2013;37(2):183-90.

10. Joiner ER, Andras LM, Skaggs DL. Screening for hip dysplasia in congenital muscular torticollis: is physical exam enough? J Child Orthop 2014;8(2):115-9.

11. Argandoña JE, Quiñones LP.Tortícolis congénita: consideraciones craneofaciales en relación a un caso clínico. Rev Dent Chile 2004;95(2):18-22.

12. Arslan H, Gündüz S, Subaşi M, Kesemenli C, Necmioğlu S. Frontal cephalometric analysis in the evaluation of facial asymmetry in torticollis, and outcomes of bipolar release in patients over 6 years of age. Arch Orthop Trauma Surg 2002;122(9-10):489-93.

13. Seo SJ, Yim SY, Lee IJ, Han DH, Kim CS, Lim Het al. Is craniofacial asymmetry progressive in untreated congenital muscular torticollis? Plast Reconstr Surg 2013;132(2):407-13.

14. Yu CC, Wong FH, Lo LJ, Chen YR. Craniofacial deformity in patients with uncorrected congenital muscular torticollis: an assessment from three-dimensional computed tomography imaging. Plast Reconstr Surg 2004;113(1):24-33.

15. Hollier L, Kim J, Grayson BH, McCarthy JG. Congenital muscular torticollis and the associated craniofacial changes.Plast Reconstr Surg 2000; 105(3):827-35.

16. Chate RA. Facial scoliosis from sternocleidomastoid torticollis: long-term postoperative evaluation. Br J Oral Maxillofac Surg 2005;43(5):428-34.

17. Kawamoto HK, Kim SS, Jarrahy R, Bradley JP. Differential diagnosis of the idiopathic laterally deviated mandible. Plast Reconstr Surg 2009;124(5):1599-609.
18. Putman GD, Postlethwaite KR, Chate RA, Ilankovan V. Facial scoliosis, a diagnostic dilemma. Int J Oral Maxillofac Surg 1993;22(6):324-7.

19. Chate RA. Facial scoliosis due to sternocleidomastoid torticollis: a cephalometric analysis. Int J Oral Maxillofac Surg 2004;33(4):338-43.

20. Joyce MB, de Chalain TM. Treatment of recalcitrant idiopathic muscular torticollis in infants with botulinum toxin type a. J Craniofac Surg 2005;16(2):321-7.

21. Nilesh K, Mukherji S. Congenital muscular torticollis. Ann Maxillofac Surg 2013;3(2):198-200.

22. Bouchard M, Chouinard S, Suchowersky O. Adult cases of congenital muscular torticollis successfully treated with botulinum toxin. Mov Disord 2010;25 (14):2453-6.

23. Díaz IV, Gómez D, García A, Rodríguez LM. Tortícolis muscular congénita en fase de adulto. Rehabilitación (Madrid) 2012;46(4):325-8.

24. Sudesh P, Bali K, Mootha AK, Dhillon MS. Results of bipolar release in the treatment of congenital muscular torticolis in patients older than 10 years of age. J Child Orthop 2010;4(3):227-32.

25. Chang SH, Ohtori S, Okawa A, Kawamura K, Saiki H, Nakada I et al. A surgical treatment for adult muscular torticollis. Case Rep Orthop 2013; http://dx.doi.org/ $10.1155 / 2013 / 965693$

26. Lee JK, Moon HJ, Park MS, YooWJ, Choi IH, Cho TJ. Change of craniofacial deformity after sternocleidomastoid muscle release in pediatric patients with congenital muscular torticollis. J Bone Joint Surg Am 2012;94(13):e93.

27. Shim JS, Jang HP. Operative treatment of congenital torticollis. J Bone Joint Surg Br 2008;90(7):934-9.

\section{CORRESPONDENCIA}

\author{
Adolfo Pipa Vallejo \\ Escuela de Odontología. Facultad de Medicina \\ Universidad de Oviedo
}

Correo Electrónico: pipaadolfo@uniovi.es. 